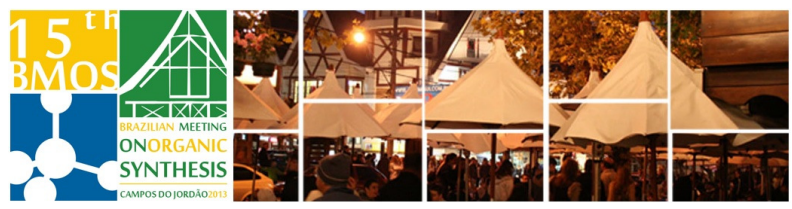

\title{
Synthesis of New Triazoles by Click Chemistry
}

\section{Cíntia M. C. F. Lima (PG), ${ }^{\star 1,2}$ Maurício M. Victor (PQ), ${ }^{1,2}$ Lenilson C. Rocha (PG), ${ }^{3}$ Alex H. Jeller (PG), ${ }^{3}$ and André L. M. Porto (PQ) ${ }^{3}$}

${ }^{1}$ Organic Chemistry Department, Chemistry Institute, and ${ }^{2}$ National Institute of Science and Technology/INCT-for Energy and Environment, UFBA, Salvador - BA, 40170-115, Brazil;

${ }^{3}$ Institute of Chemistry of São Carlos, University of São Paulo, São Carlos - SP, 13560-970, Brazil.

\section{cintialima.quimica@gmail.com}

Keywords:Triazoles, Click Chemistry, Organic Synthesis

\section{INTRODUCTION}

Click Chemistry is a Organic Synthesis procedure that follows the principles of Green Chemistry, introduced by Sharpless in 2001. ${ }^{1}$ The 1,3-dipolar copper-catalyzed azide-alkyne cycloaddition (CuAAC) is the most prominent click reaction. ${ }^{2}$ In this context, this paper aims to synthesize triazoles with potential antifungal and antileishmanial activity employing this synthetic methodology.

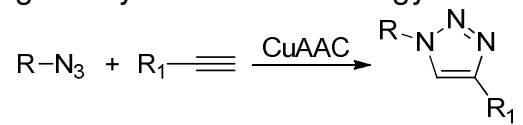

Scheme1. General structure of the triazoles.

\section{RESULTS AND DISCUSSION}

The following structures are the triazoles that were synthesized by alkynes and azides ${ }^{3}$ coupling:

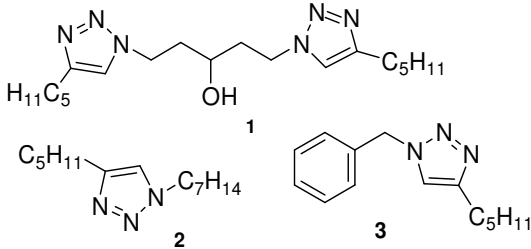<smiles>CC(=O)O[C@H](Cn1cc(COCc2ccccc2)nn1)c1ccc(Cl)cc1</smiles><smiles>[3H]OC(=O)[C@H](Cn1cc(CO)nn1)c1ccc(OC)cc1</smiles><smiles>CC(=O)O[C@H](Cn1cc(COCc2ccccc2)nn1)c1ccc(Br)cc1</smiles><smiles>CC(=O)OC[C@H]1O[C@@H](OCCc2cn(C[C@H](O)c3ccc(Br)cc3)nn2)C=C[C@@H]1OC(C)=O</smiles>

Figure 1.Triazoles synthesized.
Table 1. Compounds synthesized by Click reactions.

\begin{tabular}{|c|c|c|c|}
\hline Triazoles & $\mathbf{R}$ & $\mathbf{R}_{1}$ & $\%$ \\
\hline 1 & 1,5-diazidopentan-3-ol & hept-1-yne & 29 \\
\hline 2 & 1-azido-octane & hept-1-yne & 28 \\
\hline 3 & benzylazide & hept-1-yne & 52 \\
\hline 4 & $\begin{array}{l}\text { (S)-2-azido-1-(4-Cl- } \\
\text { phenyl)-ethyl acetate }\end{array}$ & $\begin{array}{l}\text { benzyl } \\
\text { propargyl } \\
\text { ether }\end{array}$ & 80 \\
\hline 5 & $\begin{array}{l}\text { (S)-2-azido-1-(4-Cl- } \\
\text { phenyl)ethyl acetate }\end{array}$ & hept-1-yne & 65 \\
\hline 6 & $\begin{array}{l}\text { (S)-2-azido-1-(4-Cl- } \\
\text { phenyl)ethyl acetate }\end{array}$ & $\begin{array}{l}\text { propargyl } \\
\text { alcohol }\end{array}$ & 46 \\
\hline 7 & $\begin{array}{c}(R) \text {-2-azido-1-(4- } \\
\text { methoxy-phenyl)ethanol }\end{array}$ & $\begin{array}{c}\text { benzyl } \\
\text { propargyl } \\
\text { ether }\end{array}$ & 41 \\
\hline 8 & $\begin{array}{l}\text { (S)-2-azido-1-(4-Br- } \\
\text { phenyl)ethyl acetate }\end{array}$ & $\begin{array}{c}\text { benzyl } \\
\text { propargyl } \\
\text { ether }\end{array}$ & 54 \\
\hline 9 & $\begin{array}{l}\text { (R)-2-azido-1-(4-Br- } \\
\text { phenyl)ethanol }\end{array}$ & $\begin{array}{c}\text { benzyl } \\
\text { propargyl } \\
\text { ether }\end{array}$ & 71 \\
\hline 10 & $\begin{array}{l}(R) \text {-2-azido-1-(4-Br- } \\
\text { phenyl)ethanol }\end{array}$ & sugar & 29 \\
\hline
\end{tabular}

\section{CONCLUSION}

Ten triazoles were synthesized via CuAAC, some unpublished and chiral. We will evaluate their potential antifungal and/or antileishmanial activities.

\section{ACKNOWLEDGEMENTS}

GPSQ for chemical support, Prof. Paulo H. Menezes (UFPE) for sugar derivative, and Capes for the scholarship.

\section{REFERENCES}

1 Sharpless, K.B; Finn, M.G; Kolb, H.C. Angew. Chem. Int. Ed. 2001, 40, 2004-2021.

2 Kappe, C.O; van der Eycken, E. Chem. Soc. Rev. 2009, 39,1280-1290.

${ }^{3}$ Rocha, L.C; Rosset, I. G.; Melgar, G. Z.; Raminelli, C.; Porto, A. L. M.; Jeller, A. H. J. Braz. Chem. Soc. 2013, 24, 1427-1432 